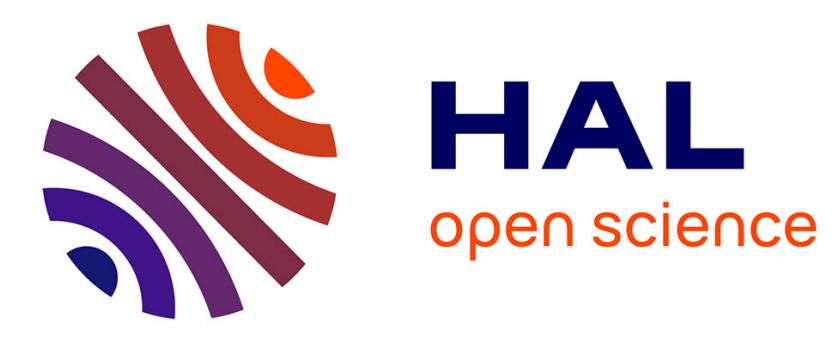

\title{
Techniques de mesures de contraintes résiduelles. Contribution à l'étude de l'amélioration de la tenue en fatigue d'alliages d'aluminium utilisés en aéronautique
}

C. Villard, A. Viola, E. Zeller, P. Castellucci, J. Duchazeaubeneix

\section{- To cite this version:}

C. Villard, A. Viola, E. Zeller, P. Castellucci, J. Duchazeaubeneix. Techniques de mesures de contraintes résiduelles. Contribution à l'étude de l'amélioration de la tenue en fatigue d'alliages d'aluminium utilisés en aéronautique. Journal de Physique IV Proceedings, 1996, 06 (C4), pp.C4-239C4-249. 10.1051/jp4:1996422 . jpa-00254305

\section{HAL Id: jpa-00254305 https://hal.science/jpa-00254305}

Submitted on 1 Jan 1996

HAL is a multi-disciplinary open access archive for the deposit and dissemination of scientific research documents, whether they are published or not. The documents may come from teaching and research institutions in France or abroad, or from public or private research centers.
L'archive ouverte pluridisciplinaire HAL, est destinée au dépôt et à la diffusion de documents scientifiques de niveau recherche, publiés ou non, émanant des établissements d'enseignement et de recherche français ou étrangers, des laboratoires publics ou privés. 


\title{
Techniques de mesures de contraintes résiduelles. Contribution à l'étude de l'amélioration de la tenue en fatigue d'alliages d'aluminium utilisés en aéronautique
}

\author{
C. Villard, A. Viola, E. Zeller*, P. Castellucci** et J.M. Duchazeaubeneix*** \\ Messier-Bugatti, 19 rue de la Commanderie, 67120 Molsheim, France \\ * IUT Génie-Mécanique, 72 route du Rhin, 67400 Illkirch-Graffenstaden, France \\ ** MECASEM, 11 Quai Heydt, BP. 47, 67540 Ostwald, France \\ *** SONATS, 2 rue Robert Schuman, 44408 Rezé cedex, France
}

\begin{abstract}
Résumé : Cette étude s'inscrit dans le cadre de la maîtrise des contraintes résiduelles de pièces en alliages d'aluminium utilisées en aéronautique. Au travers de l'exemple d'application du traitement de précontrainte par galetage des demi-roues, les difficultés rencontrées pour évaluer les contraintes résiduelles dans les pièces réelles à géométrie complexe sont abordées. La méthode du trou incrémentale pour mesurer les contraintes résiduelles est comparée avec la méthode de diffraction des rayons X. Malgré les difficultés liées aux changements d'échelles, une étude paramétrique du procédè de galetage a été menée sur éprouvettes normalisées afin d'aboutir à une caractérisation du comportement en fatigue en traction ondulée.
\end{abstract}

\begin{abstract}
This study takes place in a broader theme concerning the control of residual stresses in parts made of aluminum alloys in the aeronautical field. Through the example of stress roll operation, the difficulties in measuring residual stresses in real parts with complex shapes are presented. The hole drilling strain gagemethod is compared with the $\mathrm{X}$-ray diffraction method. In spite of the limitations inherent to scaling, the parameters influencing the stress roll process were inventoried and their effets quntified on normalised specimens to characterize the fatigue behaviour of stress rolled specimens.
\end{abstract}

\section{INTRODUCTION}

De la conception à la réalisation de pièces aéronautiques, la mesure et la maîtrise des contraintes residuelles est devenue un thème de recherche important.

La connaissance de leur influence sur la tenue en fatigue et sur les niveaux de déformations consécutives aux opérations d'usinage a conduit à de nombreuses études associées à l'amélioration des outils de mesure[1].

L'origine mécanique et thermique des contraintes résiduelles étant les causes les plus fréquentes de leur genèse au sein des alliages d'aluminium[2], elles ont aussi été les plus étudiées [3]. Nous nous proposons ici d'illustrer une démarche caractéristique de celle que nous souhaitons mener à bien en ne négligeant pas l'aspect mesure vu du côté de l'industriel, appliqué à un produit aéronautique, la $1 / 2$ roue du train avant $\mathrm{A} 330$ (fíg. 1). 


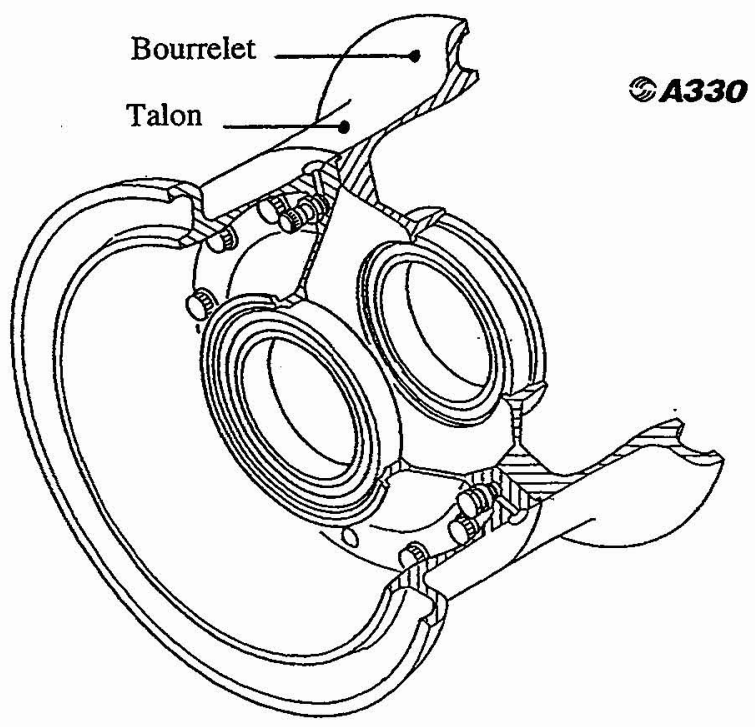

Fig. 1: Schéma de la roue du train avant A330.

Schematic of A330 nose landing gear wheel.

\section{MISE EN OEUVRE}

Pour obtenir le matricé capable d'une $1 / 2$ roue, on part d'une billette coulée en alliage 2214 (Tableau 1) qui subit une succession d'opérations de mise en forme par forgeage et matriçage. A ce stade de la réalisation, l'emploi du logiciel Forge II (TRANSVALOR) permet d'optimiser les écoulements, le fibrage et les taux de corroyage.

Les caractéristiques mécaniques d'utilisation sont obtenues par traitement thermique de mise en solution suivie d'une trempe à l'eau avec additifs puis d'un revenu (Tableau II).

Tableau I : $\quad$ Composition chimique de l'alliage 2214.

Chemical composition of 2214 alloy.

\begin{tabular}{|c|c|c|c|c|c|c|c|}
\hline $\mathrm{Si}$ & $\mathrm{Cu}$ & $\mathrm{Mg}$ & $\mathrm{Fe}$ & $\mathrm{Mn}$ & $\mathrm{Cr}$ & $\mathrm{Zn}$ & $\mathrm{Ti}$ \\
\hline $\mathbf{0 , 5}$ & 3,9 & 0,2 & & 0,4 & & & \\
1,2 & 5,0 & 0,8 & 0,3 & 1,2 & 0,1 & 0,25 & 0,15 \\
\hline
\end{tabular}

Tableau II : $\quad$ Caractéristiques physiques de l'alliage 2214 T6.

Physical characteristics of 2214 T6 alloy.

\begin{tabular}{|c|c|c|c|c|c|}
\hline $\begin{array}{c}\text { Valeurs } \\
\text { minimales } \\
\text { garanties }\end{array}$ & $\begin{array}{c}\text { Résistance à } \\
\text { rupture }\end{array}$ & Limite d'élasticité & Allongement & $\begin{array}{c}\text { Module } \\
\text { d'Young }\end{array}$ & $\begin{array}{c}\text { Coef. de } \\
\text { Poisson }\end{array}$ \\
\hline $2214 \mathrm{~T} 6$ & $\mathrm{Rr} \geq 450 \mathrm{MPa}$ & $\mathrm{Rp} 0,2 \geq 380 \mathrm{MPa}$ & $\mathrm{A} \%>6$ & $72500 \mathrm{MPa}$ & 0.33 \\
\hline
\end{tabular}


Le traitement thermique est optimisé pour permettre simultanément l'obtention des meilleures caractéristiques mécaniques et la maîtrise des contraintes résiduelles. Un modèle mathématique en 2D [4] développé en collaboration par Pechiney et Fortech apporte une contribution essentielle notamment dans la mise au point de procédés de trempe par le contrôle des contraintes résiduelles (Fig. 2). Cependant, pour être validé, ce modèle doit être confronté aux résultats des mesures de contraintes résiduelles, et pose alors le problème du choix de la méthode de mesure utilisée.

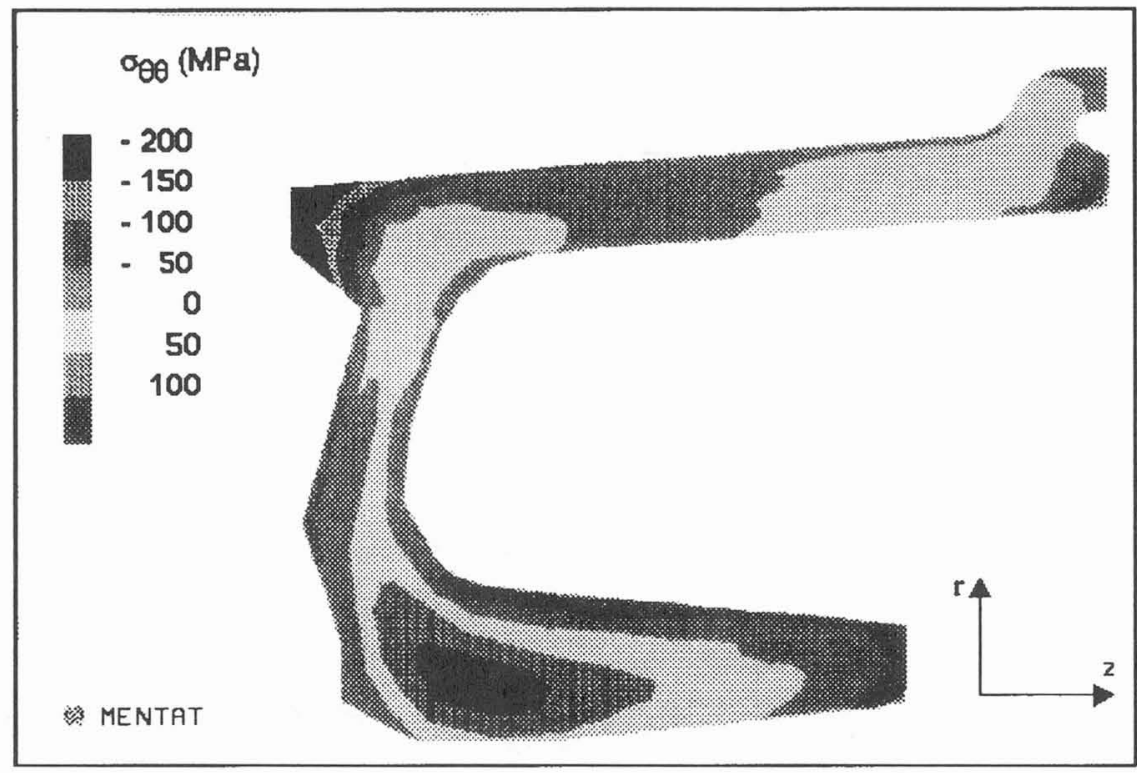

Fig. 2 : Contraintes résiduelles $\sigma \theta \theta$ calculées dans une section de demi-roue après trempe.

Section showing computed residual stresses $\sigma \theta \theta$ in wheel half after quenching.

Les alliages d'aluminium offrent un choix étendu de propriétés, et nous savons que l'obtention d'alliages à très hautes caractéristiques mécaniques s'accompagne d'une grande sensibilité aux agressions du milieu environnant. Aussi, pour assurer une protection efficace pendant une durée équivalente à 10.000 atterrissages, on réalise sur ce type de pièce une oxydation anodique qui conduit à un abattement des caractéristiques d'endurance en fatigue. Pour lutter contre cette diminution d'endurance, on a recours aux traitements mécaniques conventionnels de mise en précontrainte des surfaces. Le plus connu d'entre eux, le grenaillage de précontrainte a fait l'objet de nombreuses études [5]. Sa réalisation à l'aide de billes de céramiques [6], récemment mis au point spécifiquement pour cet alliage (Tableau III), permet de moduler les baisses évoquées. Il est aujourd'hui le traitement de précontrainte de référence appliqué aux $1 / 2$ roues, et reste très largement appliqué en aéronautique pour tous types de pièces.

La zone de la roue qui est en contact avec le pneu fait l'objet de spécification très précise pour des raisons de sécurité notamment. Pour améliorer les caractéristiques d'endurance en fatigue dans cette zone (Fig. 1), nous avons mis au point le galetage de précontrainte en substitution au grenaillage, compte tenu du fait que la géométrie de révolution se prête particulièrement bien à ce type de traitement mécanique. Les données relatives à la mise en oeuvre de ce procédé figurent au tableau IV. 
Tableau III : Conditions de grenaillage de précontrainte. Shot peening conditions.

\begin{tabular}{|c|c|c|c|c|}
\hline Machine & Nature du média & Calibre & Intensité & Tx de recouvrement \\
\hline $\begin{array}{c}\text { C.N. à } \\
\text { surpression }\end{array}$ & $\begin{array}{c}\text { Bille de } \\
\text { céramique }\end{array}$ & BC 425 & $<30 \mathrm{~N}$ & $>125 \%$ \\
\hline
\end{tabular}

Tableau IV : Conditions de galetage.

Stress rolling conditions.

\begin{tabular}{|c|c|c|c|}
\hline Machine & Principe & Galet & Paramètres \\
\hline $\begin{array}{c}\text { Tour vertical à } \\
\text { C.N. }\end{array}$ & Lame de flexion & $\begin{array}{c}\varnothing>50 \mathrm{~mm} \text { à faible } \\
\text { rayon }\end{array}$ & $\begin{array}{c}\text { Adaptation spécifique } \\
\text { au 2214 T6 }\end{array}$ \\
\hline
\end{tabular}

\section{TECHNIQUES EXPERIMENTALES}

Les mesures de contraintes résiduelles ont été réalisées par diffraction des rayons $\mathrm{X}$ et par la méthode du trou incrémentale.

Le diffractomètre mis en ceuvre est de type AST X2001 portable équipé de deux détecteurs $\mathrm{CCD}$ avec une géométrie $\psi$ modifiée (Fig. 3 et 4). En utilisant la radiation Ka du chrome, une oscillation $\psi$ systématique (angles $\psi$ à $\pm 45^{\circ}$ ) et une oscillation $\varphi$ éventuelle, il a été possible de s'affranchir des spécificités cristallographiques de l'alliage 2214.
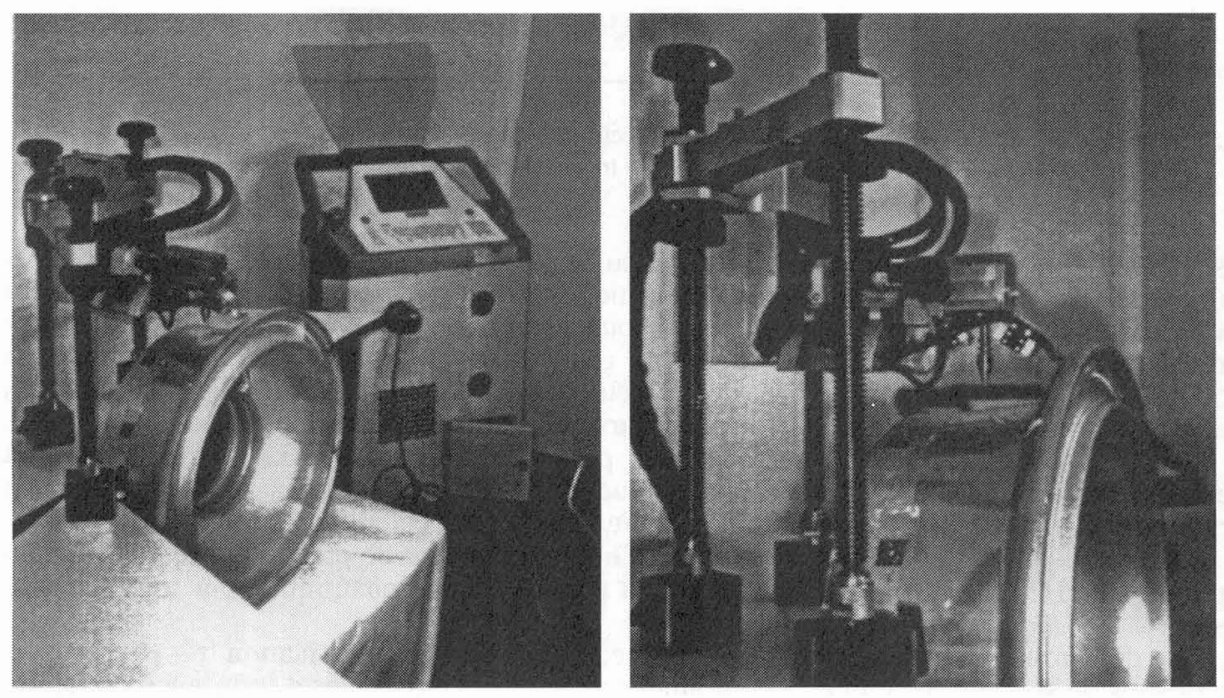

Fig. 3 et 4: Appareillage utilisé pour la mesure des contraintes résiduelles réalisée par diffraction $\mathrm{X}$ sur une demi-roue.

Equipment used to measure residual stresses by $\mathrm{X}$-ray diffraction on a wheel half. 
Les résultats présentés sont moyennés sur 3 mesures vérifiant la loi des $\sin ^{2} \psi$ avec 8 inclinaisons d'angles $\psi$ de 0 à $-45^{\circ}$ et 8 inclinaisons de 0 à $+45^{\circ}$. La méthode de détermination des positions des pics de diffraction est la Cross Corrélation [7].

Les gradients de contraintes résiduelles sont obtenus après usinages chimiques successifs. La correction de polissage sur éprouvette prélevée sur une roue a été exécutée selon la méthode de Sikarskie (norme SAE J784a) à partir d'hypothèses géométriques.

La méthode du trou incrémentale a été mise en oeuvre en utilisant des rosettes spécialement adaptées aux alliages d'aluminium. Les diamètres de perçage sont de 2 ou $4 \mathrm{~mm}$. Les calculs des contraintes résiduelles sont réalisés, soit à l'aide du logiciel METRO du Cetim, soit du logiciel RESTRESS méthode TN503-4 de Vishay-Micromesures.

La caractérisation en fatigue axiale a été réalisée à partir déprouvettes de type FCL 100 $(\mathrm{K} t=1,035)$ sollicitées en traction ondulée sur machine à résonance électromagnétique avec un rapport $\mathrm{R}=0,1$. La représentation graphique est réalisée à l'aide du modèle Onera.

L'étude fractographique a été réalisée par microscopie électronique à balayage.

\section{ETUDE}

La mise au point du galetage sur la $1 / 2$ roue a été réalisée en s'appuyant sur notre expérience des procédés de mise en précontrainte. Par la mesure des états de surface et des profils caractéristiques des états de contraintes obtenus, le procédé a pu être optimisé.

Les profils d'états de surface présentés sur la figure 5 montrent que l'une des qualité du galetage réside dans la très nette amélioration apportée par rapport à l'usinage et surtout par rapport au grenaillage.

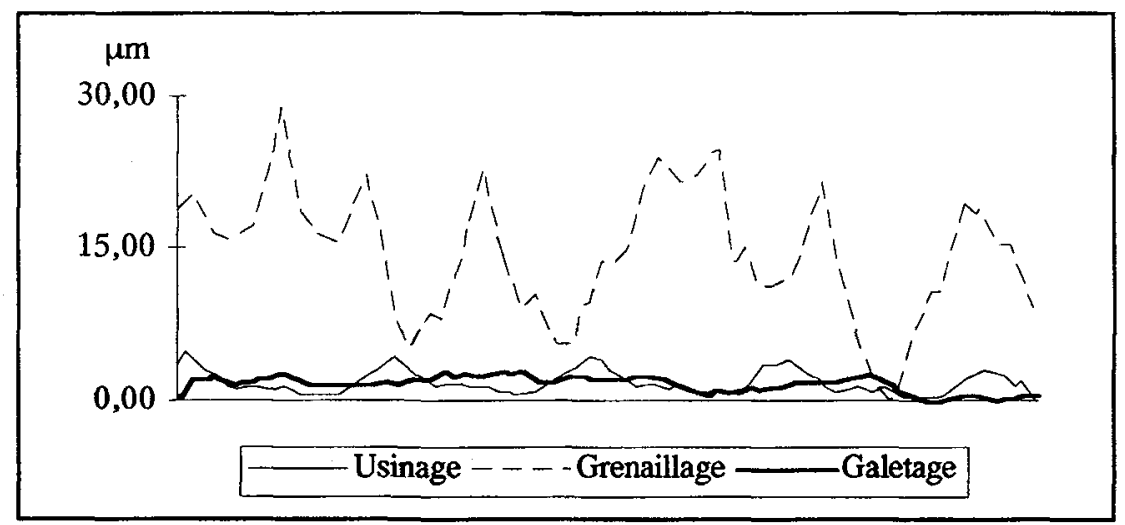

Fig. 5 : Profils d'états de surface après usinage, grenaillage ou galetage.

Surface profiles after machining, shot peening or stress rolling.

En agissant de manière précise sur certains paramètres de galetage, il est possible d'obtenir des profils de contraintes résiduelles très différents. Nous avons ainsi pu isoler des conditions de galetage industriellement acceptables qui associent un excellent état de surface et des contraintes résiduelles affectant une profondeur de 0,6 à $0,7 \mathrm{~mm}$ (figure 6). 


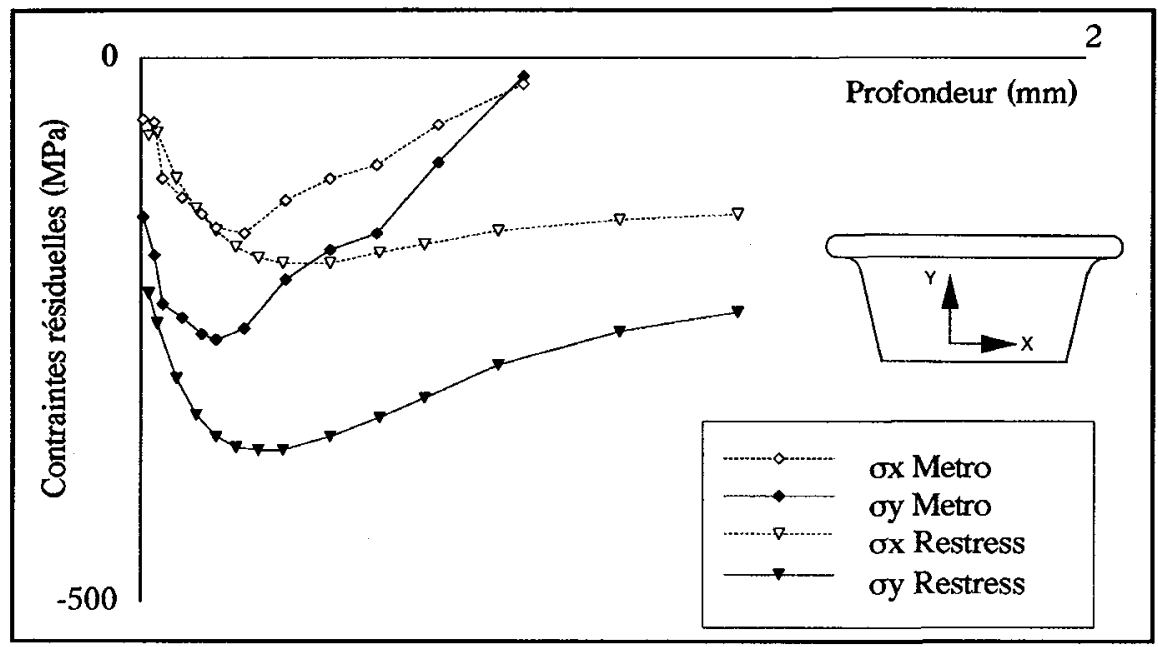

Fig. 6 : Profils de contraintes résiduelles mesurées sur une demi-roue galetée par la méthode du trou incrémentale.

Residual stresses profiles measured on a stress rolled wheel half using the hole drilling strain gage method.

Ces profils se distinguent très nettement de ceux obtenus après grenaillage.

Les résultats de' mesures des contraintes résiduelles par la méthode du trou incrémentale montrent que les contraintes résiduelles axiales $(\sigma y)$ ou circonférentielles ( $\sigma x)$ sont des contraintes résiduelles de compression avec une nette différence d'intensité. Leur répartition hétérotrophe est une conséquence de la géométrie du galet par rapport à la surface à galeter. Les deux calculs conduisent à des niveaux de contraintes différents sur tout le profil. Les différences observées en surface sont dues aux coefficients utilisés dans les deux calculs. En sous couche, l'absence de contraintes résiduelles de traction avec le logiciel RESTRESS est à attribuer au fait qu'avec ce logiciel, le calcul ne prend pas en compte le champ de contraintes résiduelles précédement relaxé à chaque incrément de perçage, contrairement au calcul du logiciel METRO.

Nous constatons que les incertitudes relatives aux premiers incréments de perçage et les écarts enregistrés avec les deux méthodes de calculs ne nous permettent pas de caractériser les profils obtenus, tant du point de vue des contraintes superficielles que de celui des contraintes maximales ou de la profondeur affectée.

L'absence de corrélation entre ces deux calculs et les difficultés d'accessibilité de l'outil de perçage dans certaines zones nous ont conduit à choisir la diffraction des rayons $\mathbf{X}$ comme méthode de mesure des contraintes résiduelles sur des surfaces possédant en plus une courbure prononcée.

Nous avons donc réalisé un profil de contraintes résiduelles par diffraction des rayons X sur une demi-roue galetée (fig. 7). 


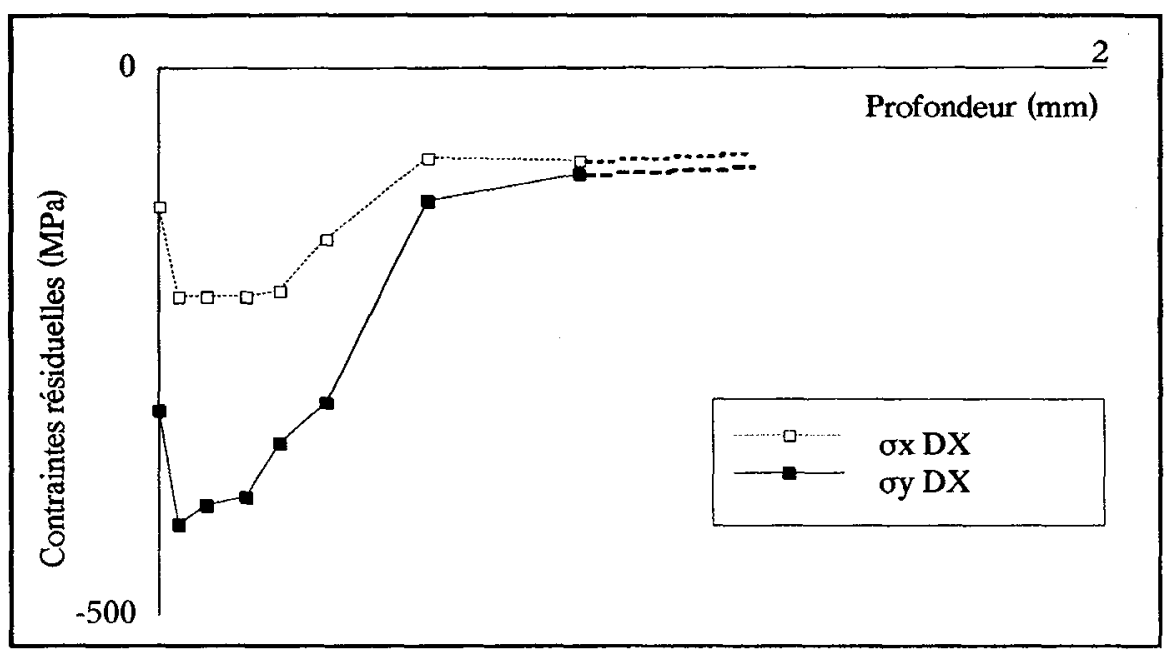

Fig. 7 : Profils de contraintes résiduelles mesurées sur une demi-roue galetée par diffraction X.

Residual stresses profiles measured on a stress rolled wheel half using X-ray diffraction method.

Ces résultats ont été obtenus après dissolutions chimiques successives appliquées à toute la pièce. On notera que des profils similaires ont été mesurés après dissolution d'une zone locale.

Les analyses réalisées nous ont confirmé le caractère hétérotrophe du champ de contraintes induit et nous ont permis d'apprécier les valeurs des contraintes résiduelles superficielles.

Nous avons ainsi pu mettre en évidence des différences sensibles entre la diffraction $\mathrm{X}$ et les méthodes du trou. En effet, en suface, les contraintes résiduelles de compression sont plus élevées avec la méthode de diffraction X, et en sous couche, elles se situent dans des valeurs intermédiaires par rapport aux deux méthodes du trou incrémentales.

Si les méthodes "mécaniques" sont aisées dans leur mise en oeuvre, elles conduisent à des résultats peu cohérents. Pour la diffraction X, les analyses effectuées révèlent la prise en compte de champs de contraintes d'origines diverses probablement liés à la forme torique de la pièce. Ceci nous a conduit à ne mettre en évidence que des contraintes de compression en sous couche bien au delà des profondeurs affectées par le galetage, et par voie de conséquence, à définir une des limitations de cette technique. Sur des pièces aussi complexes, il est en effet difficile de mener à bien une correction de polissage pour que soit pris en compte la redistribution des contraintes résiduelles après chaque enlèvement de matière.

Compte tenu des difficultés rencontrées sur la pièce réelle, nous avons refait les mesures sur un échantillon prélevé dans une demi-roue. La géométrie de œt élément assimilée à un parallélépipède, nous a permis de faire la correction de polissage selon la méthode de Sikarskie pour réaliser les profils de contraintes résiduelles et de s'affranchir ainsi du phénomène de relaxation des contraintes. Les résultats sont présentés sur la figure 8 . 


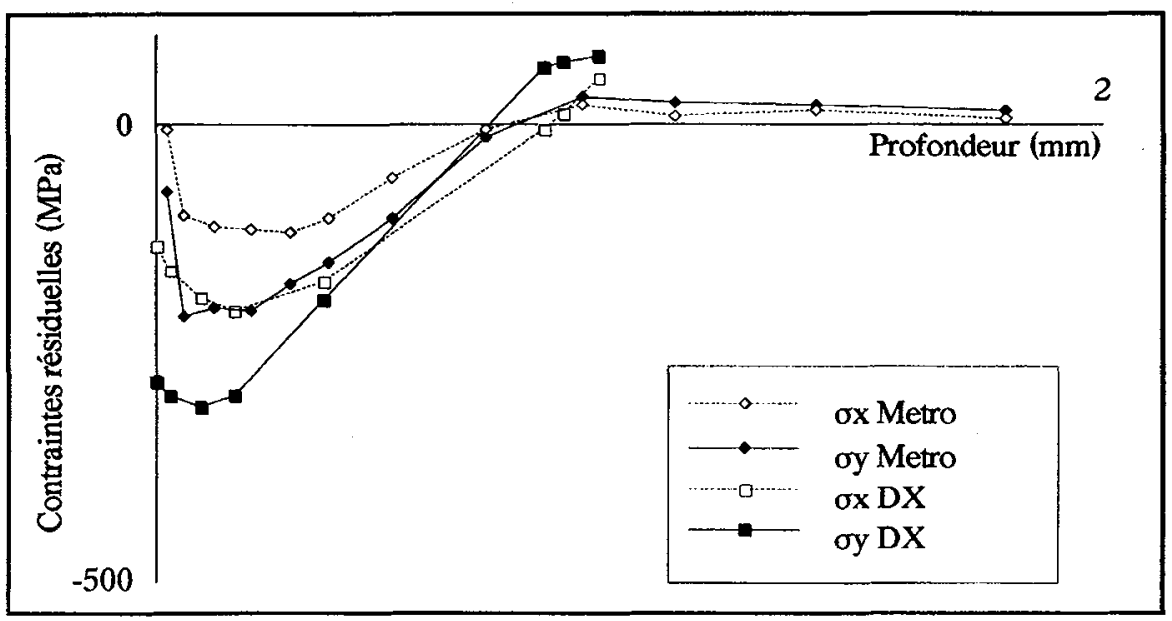

Fig. 8: Profils de contraintes résiduelles mesurées sur un échantillon de roue galetée par diffraction $\mathrm{X}$ et la méthode du trou incrémentale.

Residual stresses profiles measured on a part of stress rolled wheel half using X-ray diffraction and hole drilling strain gage methods.

Nous constatons que par rapport aux mesures effectuées sur la demi-roue entière, les contraintes résiduelles sont d'un niveau légèrement plus faibles et repassent en traction en sous couche.

Les mesures effectuées par la méthode du trou incrémentale (CETIM METRO) sur des éléments identiques ont donné ici encore des résultats difficilement comparables en terme de niveau de contrainte. On retrouve cependant une certaine analogie entre les deux types de profils et ce notamment au niveau de la profondeur affectée par les contraintes de compression. Ces profils de contraintes résiduelles mesurées par diffraction $\mathrm{X}$ sur ces éléments nous ont servi de référence.

A ce stade de notre étude et à partir de ces analyses, les objectifs ont été d'une part de pouvoir apprécier les performances en fatigue de pièces galetées en comparaison avec le grenaillage et d'autre part de valider une démarche de contrôle qualité de la production par mesures des contraintes résiduelles superficielles.

Devant l'impossibilité de pouvoir solliciter en fatigue une $1 / 2$ roue, nous avons été amenés à réaliser la caractérisation sur des éprouvettes normalisées, à géométrie modifiée pour les besoins de l'étude. Nous avons ainsi cherché à introduire lors de la réalisation du galetage des éprouvettes, les mêmes contraintes résiduelles que celles de référence.

L'adaptation du galetage s'est heurtée à de logiques mais importants changements d'échelles tant au niveau de l'outillage que de la géométrie des contacts galet-pièce. En effet, on peut considérer la $1 / 2$ roue comme étant un massif de type semi-infini devant l'effort de sollicitation lors du galetage, contrairement à l'éprouvette choisie.

Néanmoins, au cours de cette étape nous avons mené à bien une étude paramétrique complète du procédé de galetage. L'identification des paramètres fondamentaux du procédé en relation avec les niveaux de contraintes résiduelles, mesurés par diffraction $X$, nous a permi de réaliser sur éprouvettes les profils souhaités. Des profils proches de ceux dits de "référence" ont ainsi pu être obtenus (Fig. 9). 


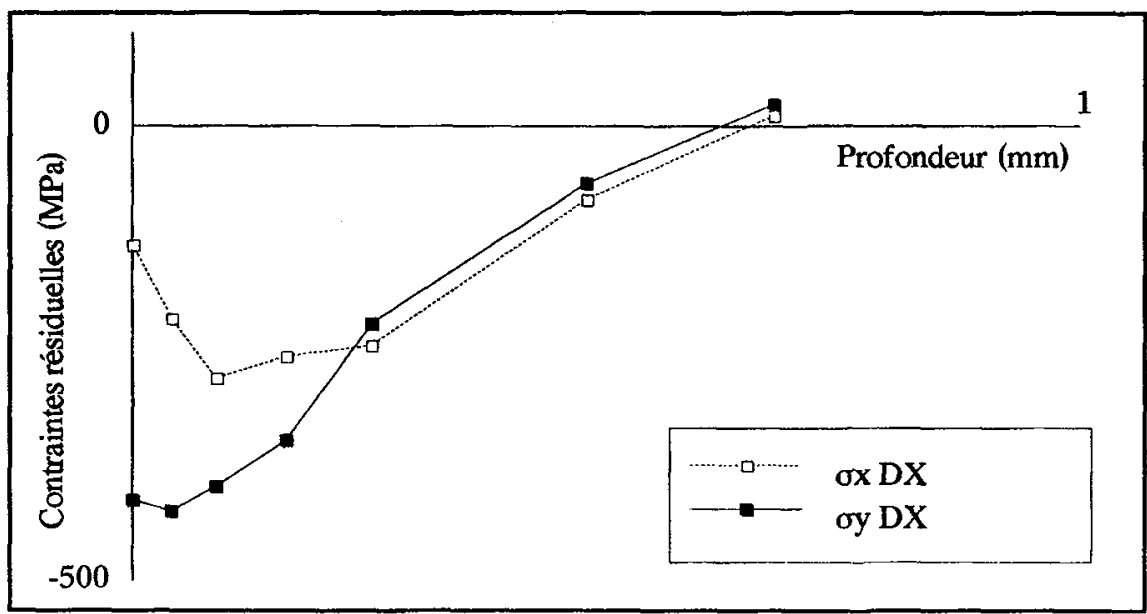

Fig. 9: Profils de contraintes résiduelles obtenus sur éprouvette galetée par diffraction X.

Residual stresses profiles measured on a stress rolled samples using X-ray diffraction method.

\section{Résistance à la fatigue}

La caractérisation complète sur éprouvettes nous a permis de montrer que les comportements en fatigue du matériau grenaillé et/ou galeté sont très différents.

Si les résultats obtenus après grenaillage sont conformes, en revanche après galetage les ébauches des courbes de Wöhler ont une allure pour le moins atypique entre $10^{5}$ et $10^{6}$ cycles (Fig. 10).

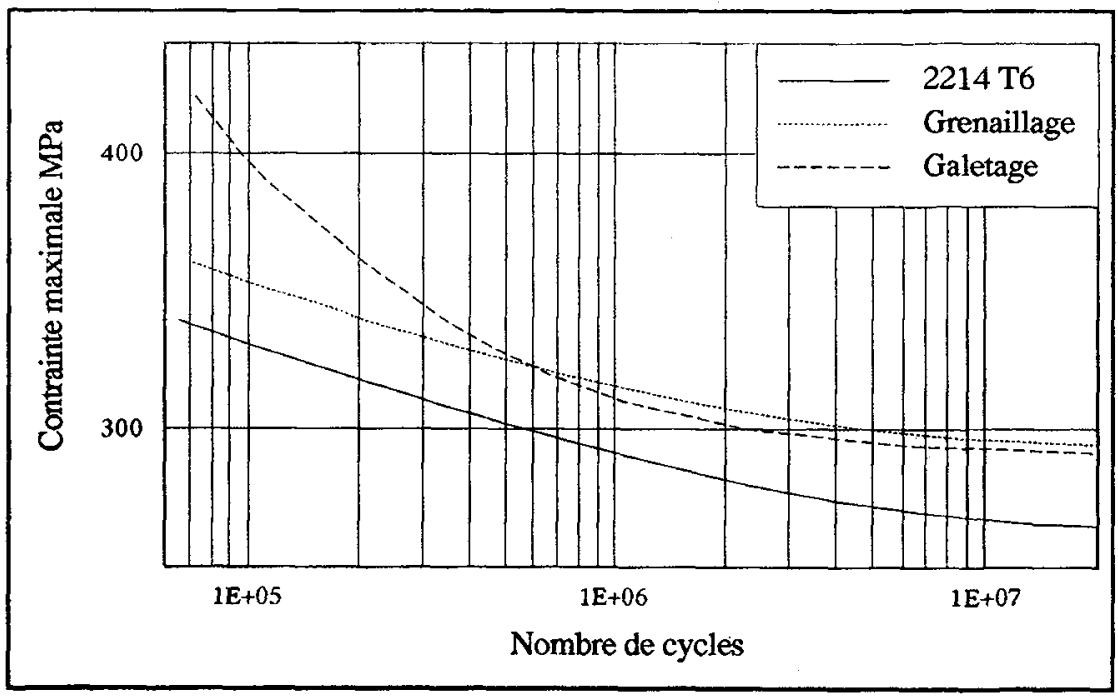

Fig. 10 : Ebauches de courbes de Wöhler après essais de fatigue sur éprouvettes FCL 100 (2214 T6 - f $\Rightarrow 5$ Hz $\mathrm{Kt}=1,035-\mathrm{R}=0,1)$.

Draft of S/N curves based on fatigue samples FCL $100(2214 \mathrm{~T} 6-\mathrm{f}=95 \mathrm{~Hz}-\mathrm{Kt}=1,035-\mathrm{R}=0,1)$ 
En effet, pour ce type de sollicitation et pour un nombre de cycles inférieurs à $10^{6}$, le modèle Onera conduit à donner à la courbe une allure quasi asymptotique où les contraintes maximales admissibles sont sensiblement plus élevées que pour le grenaillage. En revanche, au-delà de $10^{6}$ cycles et notamment à la limite d'endurance théorique à $10^{7}$ cycles, les niveaux sont proches, voire plus faibles que ceux du matériau grenaillé.

11 est vraisemblable que la sollicitation en traction ondulée $(R=0,1)$, la géométrie des éprouvettes utilisées et le profil de contraintes résiduelles résultant du galetage conduisent à ce type de comportement en privilégiant certains phénomènes d'amorçage. De plus, la présence de contraintes de traction de niveaux probablement importants à coeur qui s'additionnent à des contraintes radiales de même signe [8] y contribue. Cette analyse est à rapprocher des observations réalisées en microscopie électronique à balayage (Fig. 11).

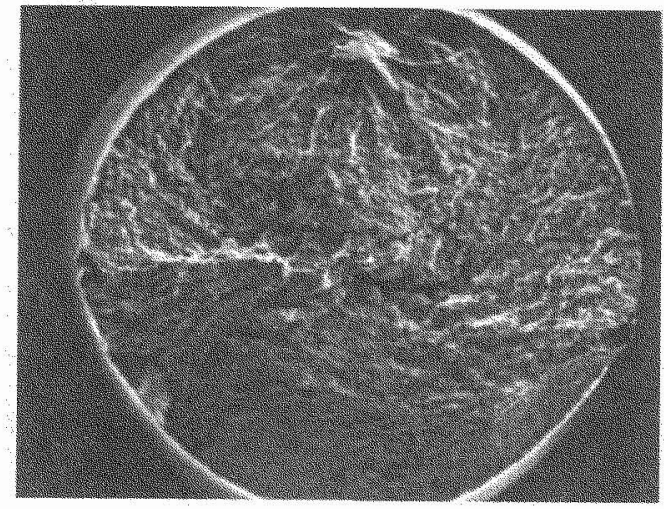

Fig. 11 : Microfractographie de la surface de rupture après essai de fatigue. Microfractograph of the fracture surface after a fatigue test.

Un calcul par éléments finis utilisant le critère de Dang Van ou de Crossland nous a confirmé qu'en présence de contraintes résiduelles de galetage, l'amorçage a lieu en sous couche.

Une évaluation de la relaxation des contraintes résiduelles au cours des essais de fatigue devrait permettre d'apporter une explication à ces différences de comportement entre le galetage et le grenaillage. Par ailleurs, il est important de souligner que le modèle ONERA a été appliqué dans un intervalle restreint de cycles et qu'il est nécessaire d'effectuer des essais complémentaires pour des chargements à contraintes imposées plus élevées.

L'étude paramétrique nous permet de constater que la variation des paramètres de galetage conduit à une modification des contraintes résiduelles superficielles. Nous avons ainsi pu définir des limites de contraintes résiduelles superficielles admissibles pour lesquels les comportements en fatigue sont similaires à $10^{7}$ cycles. Ceci va nous permettre de mettre en oeuvre la mesure superficielle des contraintes résiduelles par diffraction $\mathbf{X}$ pour le contrôle qualité de la production.

\section{CONCLUSION}

L'étude du galetage de précontrainte mis au point chez Messier-Bugatti a franchi une première étape et a atteint une partie de ses objectifs notamment par l'amélioration des états de surface et l'augmentation du niveau et de la profondeur des contraintes résiduelles. Les résultats obtenus sont particulièrement encourageants, et nous ont permis de mettre en évidence les difficultés lieées à la caractérisation des procédés industriels principalement en raison des 
facteurs d'échelles. Nos travaux se poursuivent actuellement par une caractérisation sur la tolérance aux dommages et par une modification des conditions de galetage sur éprouvettes. Enfin, avec la modélisation du procédé notamment du point de vue du contact galet/surface, et avec la maîtrise des paramètres du procédé acquise lors de cette étude, nous devrions être capable d'adapter et d'homogénéiser les champs de contraintes induits aux surfaces des matériaux à galeter.

Du point de vue de la mesure des contraintes résiduelles, les méthodes mises en oeuvre ont conduit à des résultats sensiblement différents. Si la méthode du trou incrémentale est la plus adaptée au milieu industriel, elle est parfaitement inappropriée aux mesures superficielles et parfois incohérente au niveau des profils obtenus. A l'opposé, la diffraction $X$ paraît être la plus proche de la réalité physique mais des efforts d'industrialisation reste à faire de manière générale. Au travers de ces différences, et malgré l'absence de corrélation, il nous est apparu évident que ces méthodes sont complémentaires et qu'elles constitueraient à terme le binôme analytique incontoumable pour réaliser des profils de contraintes résiduelles.

Au cours de cette étude, nous avons montré que l'évolution des paramètres conduisent à des variations de contraintes résiduelles superficielles et à des modifications de leur profil. Toutefois, dans ces conditions, ces évolutions n'induisent pas de comportement en fatigue différent à grand nombre de cycles. Il en ressort ainsi, une évidente possibilité de mise en ceuvre d'une procédure industrielle d'assurance qualité, limitée à la mesure des contraintes résiduelles superficielles réalisée par la diffraction $\mathrm{X}$ à l'aide d'un diffractomètre de type AST $\mathrm{X} 2001$.

Tous les progrès liés à la maîtrise des contraintes résiduelles et à la compréhension des phénomènes mis en jeu passeront nécessairement par une validation des modèles mathématiques, par l'amélioration des techniques de mesures des contraintes résiduelles et par la mise à disposition aux industriels de moyens aboutis afin de pouvoir travailler sur pièces réelles.

A cet égard, il est important que scientifiques et industriels y contribuent activement et travaillent à la mise en oeuvre de programmes de développement relatifs aux moyens de contrôles industriels des contraintes résiduelles.

\section{BIBLIOGRAPHIE}

[1] MUTOH Y., FAIR G.H., NOBLE B, and WATERHOUSE R.B., The effect of residual stresses induced by shot peening on fatigue crack propagation in two high strength aluminium alloys, Fatigue and fracture of engineering materials and structures, Vol 10, $N^{\circ} 4,1987$, p. 261 à 272 .

[2] KLOOS K.H., Eigenspannungen, Definition und Entstehungsursachen, Symp. Eigenspannungen Bad Mauheim, Ed. Deut. Gesell. Metallkde, 1979, p. 1 à 20.

[3] CORTEY M., HOMETTE M.O., MASSE C., Applications industrielles des mesures de contraintes par diffraction $X$. Etude des déformations de pièces en alliage d'aluminium en cours d'usinage, SURFAIR X, Cannes, 8, 9 et 10 juin 1994.

[4] DUBOST B., BOUET-GRIFFON M., JEANMART PH. and HOMMET M.O., Prediction and minimization of residual stresses in quenched aluminium alloy die forgings, International Conference on Residual stresses, ICRS2, Nancy 23-25 Novembre 1988.

[5] GENTIL B., DESVIGNES M., CASTEX L., Analyse des surfaces grenaillées : fissuration, rugosité et contraintes résiduelles, Matériaux et techniques, déc. 1987, Vol. 75, $\mathrm{N}^{\circ} 12$, p. 493 à 497.

[6] VIOLA A., Amélioration de la limite d'endurance d'alliage d'aluminium à haute résistance par grenaillage de précontrainte à la bille de céramique, 1994, Rapport interne non publié.

[7] KORHONEN M.A., LINDROOS V.K. and SUOMINEM L.S. Advances in X-Ray Analysis, Vol. 32, 1989.

[8] INGLEBERT G., FRELAT J., Quick analysis of inelastic structures using a simplified method, Nuclear Engineering and Design, $N^{\circ} 116,1989$, p. 281 à 291. 